\title{
RESISTÊNCIA IN VIVO DO PLASMODIUM FALCIPARUM ÀS 4-AMINOQUINOLEÍNAS E À ASSOCIAÇÃO SULFADOXINA-PIRIMETAMINA. II - ESTUDO DE IMPERATRIZ, MARANHÃO, 1983-1984
}

\author{
Afonso Dinis Costa Passos1, Carlos Hiroyuki Osanaj² e Santiago Reyes ${ }^{3}$
}

\begin{abstract}
Através da prova de 7 dias foram estudadas as respostas terapêticas de 96 pacientes com malária falciparum não grave atendidos pela SUCAM em Imperatriz, Maranhão. Esses pacientes foram distribuidos aleatoriamente em três grupos de estudo, tendo o primeiro recebido cloroquina, o segundo amodiaquina e o terceiro a associação sulfadoxina-pirimetamina. Mesmo sem evidenciar significância estatística ao nivel de $5 \%$ de probabilidade, as diferenças observadas nas respostas as 3 drogas apontam para a associação sulfadoxina-pirimetamina como a que produziu melhores resultados terapêuticos. Recomenda-se a monitorização contínua da resistência nas áreas malarigenas criticas.
\end{abstract}

Palavras chaves: Malária. Terapêutica. Resistência medicamentosa. Amazônia.

A resistência medicamentosa do Plasmodium falciparum vem se constituindo em um obstáculo considerável à implementação dos programas antimaláricos em diversas áreas do mundo ${ }^{9}$. Inicialmente verificada em relação à cloroquina ${ }^{5} 7$, o processo de resistência ampliou-se a outras drogas alternativas tais como a amodiaquina ${ }^{2}$ e associação sulfadoxinapirimetamina ${ }^{1}$ - criando dificuldades progressivamente maiores ao manejo clínico de pacientes e, especialmente, ao controle em larga escala da endemia.

Apesar de suas limitações, essas três drogas constituem ainda hoje as alternativas mais freqüentemente utilizadas pela Superintendência de Campanhas de Saúde Pública (SUCAM) nas suas atividades de rotina, razão pela qual torna-se necessária uma monitorização contínua da eficácia das mesmas nas áreas de transmissão ${ }^{6}$. Somente assim será possivel a utilização de uma droga até o seu limite de segurança em termos de efeito terapêutico, além do qual a mesma deverá ser substituída.

Em 1983/84, as Divisões de Epidemiologia e de Malária da SUCAM realizaram estudos in vivo de resistência do $P$. falciparum à cloroquina, amodiaquina e à associação sulfadoxina-pirimetamina em quatro áreas críticas de transmissão malárica no Brasil: Rondônia, Maranhão, Pará e Amazonas. Os resulta-

1. Departamento de Medicina Social da Faculdade de Medicina de Ribeirão Preto/Universidade de São Paulo, Ribeirão Preto, SP.

2. Departamento de Epidemiologia e Métodos Quantitavos em Saúde da Escola Nacional de Saúde Pública/ FIOCRUZ, Rio de Janeiro, RJ.

3. Superintendência de Campanhas de Saúde Pública/ SUCAM, Brasilia, DF.

Recebido para publicação em 7/10/86. dos obtidos em Porto Velho, Rondônia, já foram objeto de publicação anterior ${ }^{6}$. O presente trabalho refere-se à pesquisa realizada na cidade de Imperatriz, Estado do Maranhão.

\section{MATERIAL E MÉTODOS}

A população de estudo constou de 96 pacientes portadores de malária por $P$. falciparum que procuraram o posto de diagnóstico e tratamento da SUCAM, em Imperatriz, no periodo de 10/08/83 a 15/06/84. Esses pacientes faziam parte da demanda normal do referido posto (população de referência) e foram selecionados com base nos seguintes critérios de inclusão:

a) apresentar infecção única por $P$. falciparum com densidade parasitária entre $1.000 \mathrm{e}$ 10.000 formas assexuadas por $\mathrm{mm}^{3}$ de sangue, diagnosticada através da técnica da gota espessa.

b) não apresentar forma clinicamente grave.

c) permanecer próximo ao centro onde o estudo seria conduzido, por um periodo mínimo de 7 dias.

Os pacientes foram alocados ao acaso em 3 grupos, correspondendo cada um deles a um esquema de tratamento. $O$ grupo A ( 30 participantes) recebeu cloroquina na dose total de $25 \mathrm{mg} / \mathrm{kg}$ de peso, distribuída em 3 dias, segundo o esquema clássico de tratamento de caso agudo ( 4 comprimidos no 1 . dia e 3 nos dois dias seguintes, para adultos). $\mathrm{O}$ grupo $\mathrm{B}$ ( 36 participantes) recebeu amodiaquina em doses iguais às da cloroquina e ao grupo $\mathrm{C}$ ( 30 participantes) coube a associação sulfadoxina-pirimetamina, da Central de Medicamentos (CEME), na dose de dois com- 
Passos ADC, Osanai $C H$, Reyes S. Resisténcia in vivo do Plasmodium falciparum às 4-aminoquinoleinas e à associação sulfadoxina-pirimetamina. II-Estudo de Imperatriz, Maranhão, 1983-1984. Revista da Sociedade Brasileira de Medicina Tropical 20: 109-113, Abr-Jun, 1987.

primidos * no 1. dia e um no dia seguinte, para adultos. Indivíduos abaixo de $\mathbf{1 5}$ anos, nos $\mathbf{3}$ grupos, foram medicados segundo a posologia classicamente adotada pela SUCAM ${ }^{8}$. As drogas foram sempre ministradas sob o controle direto de uma pessoa envolvida na investigação.

Convencionando-se chamar de dia zero aquele em que o diagnóstico foi feito e a primeira dose do medicamento ministrada, os pacientes foram seguidos nos dias 1, 2, 3, 5 e 7, quando novas contagens de parasitas foram realizadas. Esta estratégia de acompanhamento baseou-se na prova prática padronizada de 7 dias, preconizada pela Organização Mundial da Saúde (OMS) para determinação de resistência à cloroquina ${ }^{3}$. Os resultados de tal prova são assim interpretados:

- quando não se encontram formas assexuadas de parasitas nos dias 6 e 7, os mesmos podem ser tanto sensiveis como resistentes em grau RI tardio (S/RI);

- quando formas assexuadas de parasitas desaparecem por pelo menos 2 dias mas reaparecem e se fazem presentes no dia 7, a infecção é considerada resistente em grau RI (precoce);

- quando os parasitas assexuados não desaparecem durante o seguimento, mas têm a sua contagem reduzida a $25 \%$ ou menos de seu nível inicial durante as primeiras 48 horas do tratamento, a infecção é considerada resistente em grau RII;

- quando a redução da parasitemia assexuada é menor do que $75 \%$ durante as primeiras 48 horas ou a parasitemia permanece a mesma ou aumenta, a infecção é considerada resistente em grau RIII.
Por razões de ordem operacional os pacientes não foram seguidos nos dias 4 e 6 . Por esse motivo, para a classificação de Susceptivel (S) ou Resistente Grau I (RI) levou-se em consideração a contagem parasitária do $50^{\circ}$ dia e do $70^{\circ}$ dia, ao invés do $60^{\circ}$ e do 7\%, conforme classicamente estabelecido.

As lâminas foram colhidas em duplicata, sendo uma examinada localmente por um microscopista experiente, mediante a contagem total das formas assexuadas em 100 campos e sua multiplicação por 5 , expressando-se o resultado por milimetro cúbico. As cópias, juntamente com os dados consolidados de cada paciente, foram enviadas para revisão ao Laboratório Central da SUCAM, em Brasilia. Maiores detalhes metodológicos já foram publicados anteriormente ${ }^{6}$.

As perdas em cada grupo de estudo, representadas por abandono do seguimento ( 2 no grupo $A, 3$ no B e 4 no C), agravamento do quadro clínico (1 no $B$ ), diagnóstico superposto de Plasmodium vivax (1 noC) e aparecimento de varicela (1 no B) foram repostas automaticamente mediante a inclusão do indivíduo imediatamente seguinte que comparecesse ao serviço e que satisfizesse os critérios de seleção. $O$ paciente em que ocorreu agravamento do quadro clínico foi encaminhado para tratamento alternativo usado de rotina.

\section{RESULTADOS}

Na Tabela 1 observa-se a distribuição da amostra segundo as variáveis droga utilizada, sexo e idade. Percebe-se um predomínio acentuado do sexo masculino no total da amostra e em cada grupo de estudo,

Tabela 1 - Distribuição da amostra de pacientes com malária por Plasmodium falciparum submetidos a trata mento com cloroquina, amodiaquina e sulfa-pirimetamina, segundo a droga utilizada, sexo e idade. Imperatriz, Maranhão, 1983-1984.

\begin{tabular}{|c|c|c|c|c|c|c|c|c|c|c|c|c|c|c|c|c|c|}
\hline \multirow{3}{*}{$\begin{array}{l}\text { Idade } \\
\text { (anos) }\end{array}$} & \multirow{3}{*}{$\begin{array}{r}\text { Droga } \\
\text { Sexo }\end{array}$} & \multicolumn{4}{|c|}{ Cloroquina } & \multicolumn{4}{|c|}{ Amodiaquina } & \multicolumn{4}{|c|}{ Sulfa-pirimetamina } & \multicolumn{4}{|c|}{ Total } \\
\hline & & \multicolumn{2}{|c|}{ Masculino } & \multicolumn{2}{|c|}{ Feminimo } & \multicolumn{2}{|c|}{ Masculino } & \multicolumn{2}{|c|}{ Feminino } & \multicolumn{2}{|c|}{ Masculino } & \multicolumn{2}{|c|}{ Feminino } & \multicolumn{2}{|c|}{ Masculino } & \multicolumn{2}{|c|}{ Feminino } \\
\hline & & $N o$ & $\%$ & $N o$ & $\%$ & $N^{o}$ & $\%$ & No & $\%$ & No & $\%$ & No & $\%$ & $N^{o}$ & $\%$ & No & $\%$ \\
\hline & $\leq 14$ & 1 & 3,6 & 1 & 50,0 & 1 & 3,2 & 1 & 20,0 & - & - & - & - & 2 & 2,2 & 2 & 28,6 \\
\hline & $15-24$ & 14 & 50,0 & 1 & 50,0 & 15 & 48,4 & 1 & 20,0 & 14 & 46,7 & - & - & 43 & 48,3 & 2 & 28,6 \\
\hline & $25-34$ & 5 & 17,9 & - & - & 11 & 35,5 & 1 & 20,0 & 6 & 20,0 & - & - & 22 & 24,7 & 1 & 14,3 \\
\hline & $35-44$ & 5 & 17,9 & - & - & 3 & 9,7 & 2 & 40,0 & 6 & 20,0 & - & - & 14 & 15,7 & 2 & 28,6 \\
\hline & $\geq 45$ & 3 & 10,7 & - & - & 1 & 3,2 & - & - & 4 & 13,3 & - & - & 8 & 9,0 & - & - \\
\hline Total & & 28 & 100,0 & 2 & 100,0 & 31 & 100,0 & 5 & 100,0 & 30 & 100,0 & - & - & 89 & 100,0 & 7 & 100,0 \\
\hline
\end{tabular}

(*) Cada comprimido contèm $500 \mathrm{mg}$ de sulfadoxina e $25 \mathrm{mg}$ de pirimetamina. 
Passos ADC, Osanai CH, Reyes S. Resisténcia in vivo do Plasmodium falciparum às 4-aminoquinoleinas e à associaçāo sulfadoxina-pirimetamina. II-Estudo de Imperatriz, Maranhāo, 1983-1984. Revista da Sociedade Brasileira de Medicina Tropical 20: 109-113, Abr-Jun, 1987.

com apenas duas mulheres no grupo $\mathrm{A}$, cinco no $\mathrm{B}$ e nenhuma no $\mathrm{C}$.

Em relação à variável idade observa-se que a distribuição da amostra é bastante homogènea nos três grupos, com a maior concentração de individuos situada na faixa de 15-24 anos, seguida dos grupos 25-34 e 35-44 anos. Agrupando-se os sexos e os grupos etários abaixo de 24 anos e acima de 35 anos: $\mathrm{x}_{4 \mathrm{GL}}^{2}=4,39 ; 0,25<\mathrm{p}<0,50$.

A distribuição do grau de resistência segundo a droga utilizada é mostrada na Tabela 2. Observa-se que nos três grupos a maior concentração de indivíduos se localiza no grau S/RI, especialmente entre os que receberam sulfadoxina-pirimetamina $(66,7 \%$, contra $43,3 \%$ no grupo da cloroquina e $41,7 \%$ no da amodiaquina). No grau RI distribuem-se $30 \%$ do grupo A, $19,4 \%$ do B e $20,0 \%$ do C, enquanto que no grau RII as proporções são de $26,7 \% 30,6 \%$ e 13,3\%, respectivamente. Apenas no grupo da amodiaquina verifica-se presença de resistência em grau RIII (3 individuos). $x_{4}^{2} \mathrm{GL}=7,27 ; 0,10<\mathrm{p}<0,25$. Agrupando-se $\mathrm{S} / \mathrm{RI}+\mathrm{R} 1$ e $\mathrm{R} 2+\mathrm{R3}: \quad \mathrm{x}_{2 \mathrm{GL}}^{2}=5,40$; $0,05<\mathrm{p}<0,10$.

A resposta terapêutica às três drogas nos diferentes dias de seguimento é mostrada na Figura 1. Nela se observa que no dia 1 os grupos das 4aminoquinoleínas apresentam médias parasitárias menores que o da sulfadoxina-pirimetamina e que, a partir dai, este último mostra uma velocidade de queda mais acentuada até o dia 2. Tendência oposta é apresentada pelo grupo da amodiaquina, com médias maiores que as demais a partir do dia 1 . As médias dos grupos da cloroquina e da sulfa-pirimetamina apresentam valores próximos nos dias 2 e 3 . Nos três grupos observa-se uma inflexão da curva nos últimos dias de seguimento, a qual se inicia no dia 3 para os que receberam cloroquina e no dia 5 para os grupos da

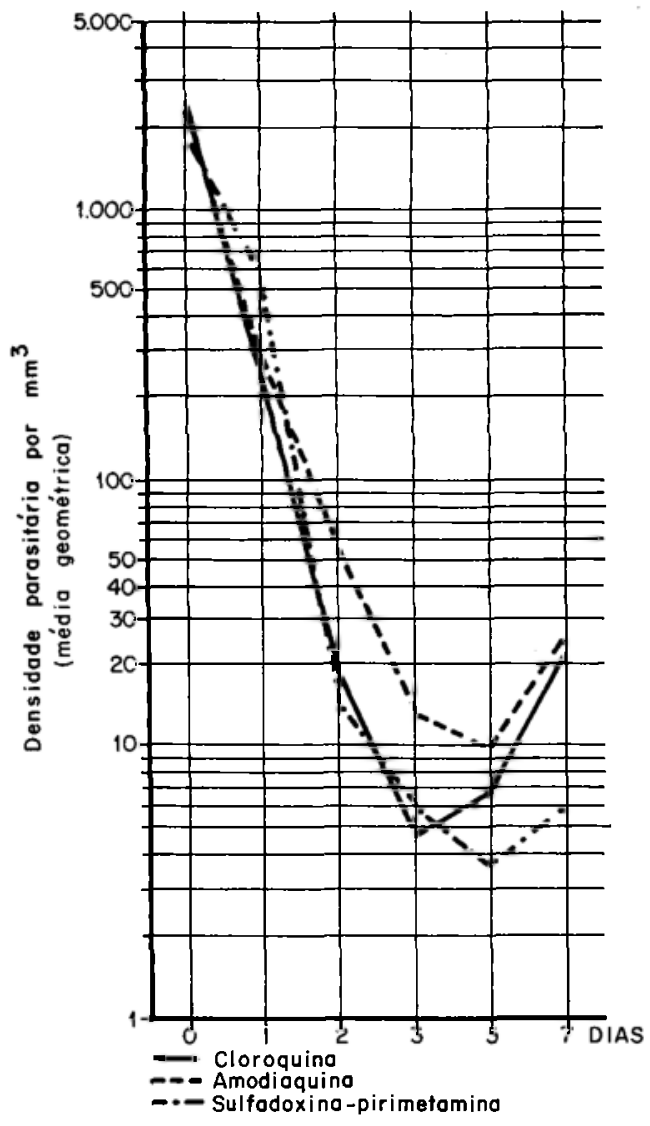

Figura 1 -Densidade parasitária de formas assexuadas de Plasmodium falciparum em individuos submetidos a tratamento com cloroquina, amodiaquina e sulfadoxinapirimetamina, segundo dias de seguimento. Imperatriz, Maranhâo, 1983-1984.

Tabela 2 - Grau de resistência do Plasmodium falciparum em pacientes submetidos a tratamento com cloroquina, amodiaquina e sulfa-pirimetamina. Imperatriz, Maranhão, 1983-1984.

\begin{tabular}{|c|c|c|c|c|c|c|c|c|c|}
\hline \multirow{2}{*}{$\begin{array}{l}\text { Grau Droga } \\
\text { de } \\
\text { Resistência }\end{array}$} & \multicolumn{3}{|c|}{ Cloroquina } & \multicolumn{3}{|c|}{ Amodiaquina } & \multicolumn{3}{|c|}{ Sulfa-pirimetamina } \\
\hline & $N .^{o}$ & $\%$ & $\% a c$ & $N .^{o}$ & $\%$ & $\% a c$ & $N^{o}$ & $\%$ & $\% a c$ \\
\hline $\mathbf{S} / \mathbf{R I}$ & 13 & 43,3 & 43,3 & 15 & 41,7 & 41,7 & 20 & 66,7 & 66,7 \\
\hline RI & 9 & 30,0 & 73,3 & 7 & 19,4 & 61,1 & 6 & 20,0 & 86,7 \\
\hline RII & 8 & 26,7 & 100,0 & 11 & 30,6 & 91,7 & 4 & 13,3 & 100,0 \\
\hline RIII & - & - & 100,0 & 3 & 8,3 & 100,0 & - & - & 100,0 \\
\hline Total & 30 & 100,0 & 100,0 & 36 & 100,0 & 100,0 & 30 & 100,0 & 100,0 \\
\hline
\end{tabular}


Passos ADC, Osanai CH, Reyes S. Resistência in vivo do Plasmodium falciparum às 4-aminoquinoleinas e à associafão sulfadoxina-pirimetamina. II-Estudo de Imperatriz, Maranhão, 1983-1984. Revista da Sociedade Brasileira de Medicina Tropical 20: 109-113, Abr-Jun, 1987.

amodiaquina e da sulfadoxina-pirimetamina. No último dia de observação as médias parasitárias nos dois grupos submetidos às 4-aminoquinoleínas mostram-se muito próximas, e em nivel acentuadamente mais alto que a média dos que receberam sulfadoxina-pirimetamina.

\section{DISCUSSÃO}

Conforme assinalado em publicação anterior ${ }^{6}$, a realização desse tipo de estudo não tem como objetivo o mapeamento do quadro de resistência medicamentosa do P. falciparum na Amazônia brasileira, para o que outra metodologia seria necessária. $O$ objetivo definido consiste no levantamento de informações que permitam aos órgãos de saúde pública orientar as suas rotinas no que diz respeito à terapêutica antimalárica, em áreas específicas. Para isso utilizou-se uma metodologia simplificada, baseada na prova de 7 dias $^{3}$, que oferece como grande vantagem a sua facilidade operacional, embora apresente a limitação de não detectar recrudescências que ocorrem após o sétimo dia. Perde-se, com isso, a possibilidade de diferenciar sensiveis de resistentes grau RI tardios, razão pela qual ambos são considerados numa mesma categoria (S/RI).

A exemplo do que ocorre em outros postos da SUCAM localizados em áreas malarigenas, também o de Imperatriz é procurado para diagnóstico e tratamento por indivíduos residentes na própria cidade, áreas vizinhas e em trânsito. Presume-se assim que o ensaio apresenta uma generalização externa razoável, permitindo extrapolar os seus resultados para a população de referência, representada pelos habitantes de Imperatriz, áreas próximas e de outras cidades com as quais Imperatriz mantém contato através de fluxo populacional constante. A análise da procedência dos pacientes, indicativa do provável local onde a infecção foi adquirida, revelou predominio das cidades de Marabá, Itaituba e São Félix do Xingu, além da própria cidade de Imperatriz, com distribuição homogênea nos 3 grupos de estudo.

Muito embora a investigação tenha sido conduzida sem um processo prévio de estratificação, observa-se uma distribuição muito semelhante quanto às variáveis sexo e idade nos três grupos de estudo, resultado do processo de casualização na alocação dos participantes.

O predominio absoluto do sexo masculino na amostra estudada, especialmente entre as idades de 15 e 34 anos, está provavelmente associado com o maior grau de exposição a que estes indivíduos são submetidos.

Ao se analisar o grau de resistência nos três grupos de estudo (Tabela 2) observa-se uma resposta melhor no grupo da sulfadoxina-pirimetamina e pior no da amodiaquina, embora com diferenças não significativas do ponto de vista estatístico. Ao se cotejarem os grupos de sensíveis e/ou resistentes em grau reduzido $(\mathrm{S} / \mathrm{RI}+\mathrm{R} 1)$ com aqueles que apresentam graus mais avançados de resistência $(\mathbf{R} 2+\mathbf{R} 3)$ essas tendências se acentuam e a análise estatistica revela um valor de probabilidade borderline $\left(\mathrm{x}_{2}^{2} \mathrm{GL}=5,40\right.$; $0,05<\mathrm{p}<0,10$ ). Fato semelhante é observado quando se analisam as proporções de individuos acima e baixo da mediana comum de parasitemia no $7{ }^{\circ}$ dia de seguimento (prova da extensão da mediana), onde se evidenciam melhores resultados com a associação sulfadoxina-pirimetamina e piores com a amodiaquina. A análise estatística mostra outra vez um valor de probabilidade limítrofe $(0,05<\mathrm{p}<0,10)$.

A aparente superioridade da sulfa-pirimetamina expressa-se também na redução da densidade parasitária nos dias de seguimento (Fig. 1), com as médias de parasitas apresentando uma inflexão mais tardia que a da cloroquina e em niveis mais baixos que os apresentados por essa e pela amodiaquina. A interpretação desta inflexão das curvas nos 3 grupos é problemática e deve ser encarada com cuidado, em virtude da limitação do ensaio a sete dias de seguimento impedir qualquer análise da evolução dos pacientes em períodos maiores.

A resposta aparentemente pior à amodiaquina que à cloroquina, discordante de alguns achados de literatura ${ }^{2}$, também exigiria uma observação mais longa para a sua correta interpretação, visto que os valores médios de parasitemia em ambos os grupos aproximam-se consideravelmente no $50^{\circ}$ e, especialmente, no $70^{\circ}$ dia.

Muito embora os dados levantados pelo presente trabalho não permitam, ao nível de $5 \%$ de significância, rejeitar a hipótese de nulidade de que não existem diferenças nas respostas de infecções por $P$. falciparum as $\mathbf{3}$ drogas testadas, observam-se resultados aparentemente melhores com o uso da associação sulfadoxina-pirimetamina, tanto no que diz respeito às menores proporçōes de graus avançados de resistência como no que se refere às mais baixas densidades parasitárias médias nos dias de seguimento. Esta situação difere da observada em estudo idêntico levado a efeito em 1983 em Porto Velho, onde foram verificadas respostas praticamente iguais nos 3 grupos, o que motivou a recomendação de que fosse mantido o uso das 4-aminoquinoleínas como droga de escolha em infecções não graves por $P$. falciparum naquela área ${ }^{6}$. Esta aparente diferença entre os dois estudos parece corroborar observações empíricas de que a resistência às 4-aminoquinoleínas ocorre de modo mais intenso na Amazônia oriental do que na ocidental. 

fadoxina-pirimetamina. II - Estudo de Imperatriz, Maranhão, 1983-1984. Revista da Sociedade Brasileira de Medicina Tropical 20: 109-113, Abr-Jun, 1987.

Apesar da impossibilidade estatistica de revelar diferenças significativas no presente estudo, os dados levantados em Imperatriz permitem recomendar o uso da associação sulfadoxina-pirimetamina em substituição às 4-aminoquinoleinas no tratamento de infecções não graves por $P$. falciparum no posto de tratamento da SUCAM naquela cidade. Merece ser destacado, todavia, que mesmo esta associação medicamentosa apresenta já um grau de efícácia comprometido, não constituindo a droga ideal no manuseio seguro de infecções por $P$. falciparum na Amazônia brasileira.

Dada a inexistência de alternativas que possam facilmente ser utilizadas a curto prazo em atividades de rotina e considerando o caráter dinâmico e variável do processo de resistência, enfatiza-se a necessidade de uma monitorização contínua do fenômeno nas áreas maláricas mais criticas.

O estabelecimento deste sistema de monitorização era um dos objetivos da Divisão de Epidemiologia da SUCAM à época da realização deste trabalho, representando o mesmo o primeiro passo visualizado e concretizado na implementação de uma ação eficaz de vigilância permanente da resistência medicamentosa do $P$. falciparum. A sua retomada, como atividade de rotina, impõe-se como fundamental na luta antimalárica no País.

\section{SUMMARY}

The in vivo drug response of 96 patients with falciparum malaria was studied to chloroquine, amodiaquine or the combination of sulfadoxine and pyrimethamine, in Imperatriz, State of Maranhão, Brasil. The patients were randomly assigned to the three study groups and followed for seven days. Better results were obtained with the association sulfadoxine-pyrimethamine, although no statistically significant differences were observed. The need of continous surveillance of $\mathbf{P}$. falciparum drug resistance in areas of high levels of transmission is emphasised.

Key words: Malaria. Chemotherapy. Resistance to drugs. Amazon basin.

\section{AGRADECIMENTOS}

Os autores agradecem aos Doutores Ernani Wilson Bezerra Carneiro e Euclides Neiva, respectivamente Diretor Regional da SUCAM no Maranhão e Chefe do Distrito de Imperatriz à época da realizaçāo do estudo e a Luiz França Smith Pereira, Maria Amélia de Morais e Maria Alice Fernandes Santos, microscopistas da SUCAM, pela colaboração na realização deste trabalho.

\section{REFERÊNCIAS BIBLIOGRÁFICAS}

1. Alecrim WD, Alecrim MGC, Dourado HC, Passos LF, Wanssa E. Resistência do $P$. falciparum à associaçāo sulfadoxina mais pirimetamina no Amazonas a nivel de RIII. In: Resumos do XVIII Congresso da Sociedade Brasileira de Medicina Tropical, Caldas Novas, 1981.

2. Hall AP, Segal HE, Pearlman EJ, Phintoyothin P, Kosakal S. Amodiaquine resistant falciparum malaria in Thailand. American Journal of Tropical Medicine and Hygiene 24:575-580, 1975.

3. Organizacion Mundial de la Salud. Quimioterapia del paludismo y resistencia a los medicamentos antipalúdicos. Série de Informes Técnicos 529: $132 \mathrm{p}$. Genebra, 1973.

4. Organizacion Panamericana de la Salud. Epidemiologia y Control de la malária causada por Plasmodium falciparum en las Américas. Washington, Publicación Cientifica no 471, 48 p. 1984.

5. Reyes S. Infecções maláricas por Plasmodium falciparum resistentes ao tratamento com cloroquina: situação no Brasil (1960-1981), Revista Brasileira de Malariologia e Doenças Tropicais 33:109-130, 1981.

6. Reyes S, Passos ADC, Osanai CH. Resistencia "in vivo" do Plasmodium falciparum às 4-aminoquinoleinas e à associação sulfadoxina-pirimetamina. I. Estudo de Porto Velho, Rondonnia, 1983. Revista da Sociedade Brasileira de Medicina Tropical 18:175-181, 1985.

7. Silva JR, Lopes PFA, Ferreira LF. Morteo R, Naveira JB. Resistência do P. falciparum à ação da cloroquina. $\mathrm{O}$ Hospital 60:581-594, 1961.

8. Superintendência de Campanhas de Saúde Pública Manual de terapêutica da malária. 82 p., Brasilia, 1982.

9. World Health Organization. Principles of malaria vaccine trials: Memorandum from a WHO meeting. Bulletin of the World Health Organization 64:185-204, 1986. 